\title{
Elevated Serum Interleukin-18 Level is Correlated with Vascular Access Dysfunction in Patients on Maintenance Haemodialysis
}

\author{
Li $\underline{\text { You }},{ }^{* 1,2}{ }_{M D}$, PhD, Yuanhao $\underline{\mathrm{Wu}},{ }^{* 1,2}{ }_{M D}$, Yin Zheng, ${ }^{1,2}{ }_{M D}$, PhD, Junfeng Liu, ${ }^{1,2}{ }_{M D}$, PhD , Jun $\underline{\mathrm{Xue}},{ }^{1,2}{ }_{M D, P h D}$
}

\begin{abstract}
Introduction: We evaluated the impact of serum interleukin-18 (IL-18) level on short-term vascular access (VA) function in chronic haemodialysis (HD) patients. Materials and Methods: Samples were collected from 80 clinically stable patients (58.8\% were men) with a mean age of 60.9 years (standard deviation 11.7 years) who were undergoing maintenance $\mathrm{HD}$ and were followed up for 1 year. Multivariate logistic regression was used to analyse data on demographics, biochemical parameters and serum IL-18 level to predict VA dysfunction events. The cut-off for IL-18 was derived from the highest score obtained on Youden index. Survival data was analysed using Cox proportional hazards regression analysis and Kaplan-Meier method. Results: Patients were classified as having either low IL-18 $(<199.3 \mathrm{pg} / \mathrm{mL})$ or high IL-18 $(\geq 199.3 \mathrm{pg} / \mathrm{mL}$ ). Multivariate logistic regression showed that serum IL-18 level was independently correlated with VA dysfunction events; patients with high IL-18 had a higher risk of VA dysfunction events than those with low IL-18 (odds ratio 9.47, 95\% confidence interval 1.75-51.31, $P=0.009$ ). In patients with high IL-18, KaplanMeier survival analysis found that incidence of VA dysfunction was significantly higher than patients with low IL-18 $(P=\mathbf{0 . 0 4 7})$. After adjustment for age, gender, inflammation (C-reactive protein) and calcium-phosphorus metabolism, decreased serum albumin and increased serum IL-18 levels were found to be independent prognostic predictors of VA dysfunction. Conclusion: HD patients with high IL-18 level tend to have worse rates of VA dysfunction. In HD outpatients, IL-18 is an independent risk factor for short-term VA dysfunction.
\end{abstract}

Ann Acad Med Singapore 2020;49:119-26

Key words: Access survival, Cytokines, Short-term

\section{Introduction}

Renal replacement therapy (RRT) is a long-term, life-sustaining intervention in patients with endstage renal disease (ESRD) and most of them are on haemodialysis (HD). ${ }^{1}$ Although various dialysis treatments have improved the prognosis in HD patients, ${ }^{2}$ the establishment and maintenance of appropriate vascular access (VA) and reduction in incidence of VA dysfunction are important factors that can impact on their long-term prognoses. ${ }^{3}$

In the last few decades, extensive application of autologous arteriovenous fistula (AVF) and innovations in VA-including arteriovenous graft (AVG) and tunnelled cuffed catheter (TCC)-have improved prognosis in HD patients. However, 9-16\% of HD patients still experience insufficient dialysis that is attributed to VA dysfunction and complications of the access point. ${ }^{4}$ Therefore, it is crucial to understand the pathogenesis of VA dysfunction, identify possible risk factors and provide early intervention.

Currently, it is believed that systemic abnormalities in ESRD patients-such as uraemic toxins, systemic inflammation, endothelial dysfunction, lipid abnormalities, hyperparathyroidism,

${ }^{1}$ Department of Nephrology, Huashan Hospital, Fudan University, People's Republic of China

${ }^{2}$ Department of Nephrology, North Huashan Hospital, Fudan University, People's Republic of China

*Both authors contributed equally to this work.

Address for Correspondence: Prof Xue Jun, Department of Nephrology, Huashan Hospital, Fudan University, No.12 Middle Wulumuqi Road, Shanghai 200040, People's Republic of China.

Email: xuejun@fudan.edu.cn 
hyperphosphataemia and hypercalcaemia — can easily remodel and narrow the vascular wall of mature AVF. ${ }^{5-7}$ In ESRD patients, some studies have found that the levels of cytokines-such as interleukin-6 (IL-6), transforming growth factor beta (TGF-beta) and tumour necrosis factor alpha (TNF-alpha) are significantly higher than those in the general population. Elevated levels of cytokines may be linked to the development of neointimal hyperplasia (NIH) and progression of VA dysfunction; however, their pathogeneses remain unclear. ${ }^{8,9}$

Interleukin-18 (IL-18) is a member of the interleukin-1 superfamily and can induce an immune response and inflammatory reaction. ${ }^{10,11}$ In patients with chronic kidney disease (CKD) and those who were on continuous ambulatory peritoneal dialysis (CAPD), studies have found evidence of chronic inflammation and increased levels of serum IL-18. ${ }^{12}$ Formanowicz et $\mathrm{a}^{13}$ have reported that IL-18 level can predict cardiovascular mortality in patients with CKD. Recently, Wang et a ${ }^{14}$ found that serum IL-18 level is an independent risk factor for major adverse cardiovascular events in HD patients.

Other studies have reported a correlation between elevated IL-18 levels and increased hospitalisation in HD patients. ${ }^{15}$ In their observational study, Liu et $\mathrm{al}^{16}$ found that IL-18 level was an independent risk factor for all-cause mortality in patients on maintenance dialysis but no correlation was found with cardiovascular function. However, it is unclear whether IL-18 contributes to the development and progression of VA dysfunction, and if it can be used as an early predictor of VA dysfunction in patients on maintenance dialysis.

Consequently, we conducted a 1-year prospective, observational study to examine the correlation between serum IL-18 level and VA function in patients who were attending our outpatient HD centre. The primary outcome of interest was incidence of VA dysfunction, and a preliminary evaluation of risk factors for VA dysfunction in patients on maintenance HD was performed.

\section{Materials and Methods}

The study commenced on 1 June 2017 and included 80 mainland Chinese patients who had been undergoing regular dialysis - 4 hours per session - thrice a week for at least 1 month in our HD centre. All of them were followed up for 1 year until 31 May 2018. Information on their medical history, primary diagnosis and complications were collected. To ensure that all patients met the criteria for adequate dialysis, they were evaluated according to the guidelines of the National Kidney Foundation Kidney Disease Outcomes Quality Initiative (NKF-KDOQI). ${ }^{17}$ The study was approved by the Institutional Review Board (IRB No.: KY2016-394) of our centre and all patients provided written consent.

The definition of VA dysfunction is based on the consensus statement on VA to HD published by the Chinese Hospital Association ${ }^{18}$ which stipulates the presence of any of the following signs and symptoms: 1) flow rate $<600 \mathrm{~mL} / \mathrm{min}$ and $<500 \mathrm{~mL} / \mathrm{min}$ for internal fistula graft and autologous internal fistula, respectively; 2) static pressure ratio of venous end of internal fistula graft or autologous internal fistula $>0.5$, or static pressure ratio of arterial end of internal fistula graft $>0.75 ; 3$ ) acute thrombosis; and 4) sufficient dialysis cannot be achieved when arterial pressure is $<250 \mathrm{mmHg}$ or venous pressure is $>250 \mathrm{mmHg}$ in the event that catheter blood flow is $<200 \mathrm{~mL} / \mathrm{min}$ or blood pump flow is $<200 \mathrm{~mL} / \mathrm{min}$.

VA dysfunction event is defined as: 1) percutaneous angioplasty or surgery to treat AVF vascular stenosis; 2) Fogarty catheter thrombectomy, surgical thrombectomy or internal fistula reconstruction to treat AVF thrombosis; and 3) balloon dilatation and stent implantation to treat AVG stenosis.

Demographic and dialysis-related data were collected including age, gender, primary disease, dialysis age, systolic blood pressure (SBP) and diastolic blood pressure (DBP) before dialysis and interval dialysis weight gain (IDWG). IDWG was derived by subtracting body weight before dialysis from body weight after previous dialysis and then dividing the result over body weight after previous dialysis.

In the middle of the week, fasting and resting venous blood was drawn from patients prior to dialysis. The blood samples were centrifuged at $12,000 \times \mathrm{g}$ at $4^{\circ} \mathrm{C}$ for 15 minutes to separate the serum from supernatant, and were stored at $-80^{\circ} \mathrm{C}$. Findings of biochemical parameters included haemoglobin $(\mathrm{Hb})$, white blood cell (WBC), platelet (PLT), creatinine, albumin (Alb), total cholesterol (CHO), low-density lipoprotein cholesterol (LDL), B-type natriuretic peptide (BNP), C-reactive protein (CRP), intact parathyroid hormone (iPTH), blood calcium (Ca) and blood phosphorus (P). To evaluate adequacy of dialysis, urea $\mathrm{Kt} / \mathrm{V}$ was used. ${ }^{17}$

Serum IL-18 was determined with enzyme-linked immunosorbent assay (Human IL-18 ELISA Kit, MyBioSource, San Diego, CA, USA). The cut-off for IL-18 was derived from the highest score obtained on Youden index. ${ }^{19}$ Patients were classified as having either low $(<199.3 \mathrm{pg} / \mathrm{mL})$ or high $(\geq 199.3 \mathrm{pg} / \mathrm{mL})$ IL-18. 
Statistical analyses were performed with Stata Statistical Software, Release 14 (StataCorp LP, College Station, TX, USA). Continuous variables were expressed as mean and standard deviation (SD) or median and interquartile range; categorical variables were expressed as counts and percentages. Student's t-test or Mann-Whitney U test (non-normal distribution) was performed to compare differences among continuous variables; to compare categorical variables, chi-square test or Fisher's Exact test was used. Univariate or multifactorial logistic regression was used to analyse correlations between biochemical markers and dialysis-related data with VA dysfunction. Kaplan-Meier method and logarithmic rank test were used to compare adverse VA events between the 2 groups. Univariate and multivariate Cox regression analyses were used to examine risk factors for VA dysfunction. A value of $P<0.05$ was considered statistically significant.

\section{Results}

There were $47(58.8 \%)$ male and $33(41.2 \%)$ female patients in the study. Their mean age was 60.93 years (SD 11.70 years, range 32-89 years). Median duration of dialysis was 3.14 years. Aetiologies of ESRD included chronic glomerulonephritis $(\mathrm{n}=32,40 \%)$, hypertensive nephropathy $(\mathrm{n}=20,25 \%)$, diabetic nephropathy $(\mathrm{n}=9,11.25 \%)$, polycystic kidney disease $(\mathrm{n}=7,8.75 \%)$ and other causes $(\mathrm{n}=12,15 \%)$. VA types included AVF $(\mathrm{n}=64,80 \%), \operatorname{AVG}(\mathrm{n}=3,3.75 \%)$ and TCC $(\mathrm{n}=13,16.25 \%)$.

There were 30 and 50 patients in the high and low IL-18 groups, respectively. In both groups, no differences were found in age, gender, dialysis age, diabetes-to-hypertension ratio, mean IDWG, SBP and DBP before dialysis $(P>0.05)$. Likewise, no differences were seen in results of biochemical parameters between the 2 groups $(P>0.05)$ including creatinine, BNP, CRP, iPTH, calcium-phosphorus product, $\mathrm{Hb}, \mathrm{WBC}, \mathrm{PLT}, \mathrm{CHO}$ and LDL.

Serum albumin level was lower in the high IL-18 $(41.6 \mathrm{~g} / \mathrm{L})$ group than low IL-18 $(43.4 \mathrm{~g} / \mathrm{L})$ group $(P=0.003)$. During the study, $11(13.75 \%)$ patients experienced VA dysfunction and required surgical intervention; 2 patients experienced VA events within half a year after access was established and the remainder had late-stage VA dysfunction. As shown in Table 1, although the incidence of VA dysfunction was higher in the high IL-18 group, the difference between the 2 groups $(23.3 \%$ vs $8 \%$ ) was not statistically significant $(P=0.973)$.
After adjustments for age, gender, dialysis age, iPTH, calcium-phosphorus product, CRP and albumin, multivariate logistic regression found that only IL-18 level was independently correlated with VA dysfunction events (Table 2). The high IL-18 group had a higher risk of VA dysfunction events than the low IL-18 group (odds ratio 9.47, 95\% confidence interval 1.75-51.31, $P=0.009)$. Kaplan-Meier survival analysis (Fig. 1) showed that VA dysfunction in the high IL-18 group was significantly higher than low IL-18 group $(P=0.047)$.

Findings of multivariate Cox proportional hazards regression model revealed that decreased serum albumin level and increased serum IL-18 level were independent prognostic predictors of VA dysfunction events (Table 3). This predictive effect was still statistically significant even after adjustments were made for age, gender, inflammation (CRP) and calcium-phosphorus metabolism (calcium-phosphorus product). Additionally, a significant correlation between serum IL-18 level and reduced albumin level for VA dysfunction events was not found $(P=0.27)$. Findings from receiver operating characteristic curve analysis showed that when IL-18 and albumin levels were used with age and gender, short-term (within 1 year) risk of VA dysfunction can be predicted in HD patients (Fig. 2).

\section{Discussion}

This prospective cohort study demonstrated that elevated serum IL-18 level is correlated with VA dysfunction in patients on maintenance HD, and that it is also an independent risk factor for short-term VA dysfunction in them. Patients with high serum IL-18 concentration also had higher incidence of VA dysfunction. Additionally, the combination of albumin, IL-18, age and gender can be a better predictor of short-term risk of VA dysfunction in HD patients.

VA is the lifeline of HD patients, ${ }^{5}$ and VA dysfunction is a major cause of insufficient dialysis that affects their long-term prognosis. ${ }^{4}$ Due to its high patency rate, lower rate of complications and lower cost, AVF is recommended as the preferred access for HD by NKF-KDOQI. ${ }^{20}$ This was clearly seen in our patients where $80 \%$ of them used AVF. In the 11 patients who experienced VA dysfunction, $4(36.4 \%)$ of them had AVF, 5 (45.5\%) had TCC and 2 (18.1\%) had AVG. The issue of lowering the incidence of VA dysfunction, especially in HD patients with short- and long-term AVF dysfunction, continues to pose a huge challenge to dialysis physicians. 
Table 1. Demographic and Clinical Characteristics of Haemodialysis Patients

\begin{tabular}{|c|c|c|c|c|}
\hline Variable & $\begin{array}{l}\text { Aggregate } \\
(\mathbf{n}=\mathbf{8 0})\end{array}$ & $\begin{array}{c}\text { IL-18 }<199.3 \mathrm{pg} / \mathrm{mL} \\
(\mathrm{n}=50)\end{array}$ & $\begin{array}{c}\text { IL-18 } \geq 199.3 \mathrm{pg} / \mathrm{mL} \\
(\mathrm{n}=30)\end{array}$ & $P$ Value \\
\hline Mean age in years (SD) & $60.9(11.7)$ & $60.3(11.9)$ & $62.0(11.5)$ & 0.731 \\
\hline Male gender $(\%)$ & $47(58.8)$ & $32(64.0)$ & $15(50.0)$ & 0.109 \\
\hline Median dialysis age in years (IQR) & $3.14(2.25-6.75)$ & $2.85(2.15-6.23)$ & $4.03(2.42-7.42)$ & 0.201 \\
\hline Diabetes mellitus (\%) & $9(11.25)$ & $6(12)$ & $3(10)$ & 0.392 \\
\hline Hypertension (\%) & $20(25)$ & $12(24)$ & $8(26.7)$ & 0.605 \\
\hline Mean IDWG, \% (SD) & $3.3(1.2)$ & $3.3(1.2)$ & $3.4(1.2)$ & 0.612 \\
\hline Median Kt/V (IQR) & $1.36(1.16-1.5)$ & $1.37(1.15-1.55)$ & $1.30(1.18-1.46)$ & 0.538 \\
\hline Mean SBP, mmHg (SD) & $142.1(15.6)$ & $143.8(14.6)$ & $139.4(16.9)$ & 0.122 \\
\hline Mean creatinine, $\mu \mathrm{mol} / \mathrm{L}(\mathrm{SD})$ & $1009.6(250.2)$ & $1014.8(258.8)$ & $1001(239.1)$ & 0.809 \\
\hline Median BNP, pg/mL (IQR) & $2658(1429-5084)$ & $2978.5(1362-5473)$ & $1962(1497-3712)$ & 0.290 \\
\hline Median albumin, g/L (IQR) & $42.2(39.8-44.0)$ & $43.4(41.1-48.7)$ & $41.6(39.2-43.1)$ & 0.003 \\
\hline Median C-reactive protein, mg/L (IQR) & $2.9(0.91-5.4)$ & $2.6(0.88-7.2)$ & $3.0(0.94-4.67)$ & 0.992 \\
\hline Median iPTH, pg/mL (IQR) & $385(180.2-639.6)$ & $353.2(180.2-596.9)$ & $390(182.6-639.6)$ & 0.936 \\
\hline Mean $\mathrm{Ca} \times \mathrm{P}, \mathrm{mg}^{2} / \mathrm{dL}^{2}(\mathrm{SD})$ & $57.4(16.8)$ & $59.5(15.8)$ & $53.9(18.2)$ & 0.083 \\
\hline Median haemoglobin, g/L (IQR) & $105.5(95.5-113)$ & $107(97-115)$ & $104(94-113)$ & 0.423 \\
\hline Median WBC count, $\times 10^{9} / \mathrm{L}(\mathrm{IQR})$ & $6.03(5.24-7.36)$ & $6.03(5.41-7.48)$ & $6.04(5.18-7.24)$ & 0.901 \\
\hline Median platelet count, $\times 10^{9} / \mathrm{L}(\mathrm{IQR})$ & $168(138-211)$ & $170(139-212)$ & $166(136-211)$ & 0.885 \\
\hline Median LDL, mmol/L (IQR) & $2.3(1.9-2.8)$ & $2.3(1.9-2.7)$ & $2.6(1.8-3.3)$ & 0.271 \\
\hline Vascular access dysfunction (\%) & $11(13.75)$ & $4(8)$ & $7(23.3)$ & 0.973 \\
\hline
\end{tabular}

BNP: B-type natriuretic peptide; Ca $\times$ P: Calcium-phosphorus product; DBP: Diastolic blood pressure; IDWG: Interval dialysis weight gain; iPTH: Immunoreactive parathyroid hormone; IL-18: Interleukin-18; IQR: Interquartile range; LDL: Low-density lipoprotein; SBP: Systolic blood pressure; SD: Standard deviation; WBC: White blood cell

The development and progression of AVF dysfunction is complex. In ESRD patients, some studies found that they often experienced systemic microinflammation of factors such as IL-6, interleukin- 8 and TNF-alpha which contribute to the development of AVF dysplasia. ${ }^{21}$ In uraemia, elevation of IL-6, TGF-beta and TNF-alpha also renders the vascular wall of mature AVF easy to remodel and narrow, and has been linked to the development of NIH and advanced AVF dysfunction. ${ }^{8,9}$ However, the specific mechanisms that contribute to these adverse events have not been elucidated.

IL-18 is produced by an inactive $24-\mathrm{kDa}$ precursor, preIL-18, that generates an $18-\mathrm{kDa}$ protein with an endoprotease IL-1-beta-converting enzyme. IL-18 promotes maturation of $\mathrm{T}$ cells and natural killer cells, as well as synthesis of cytokines and increased cytotoxicity. ${ }^{22}$ IL-18 and its receptor are expressed in endothelial cells, vascular smooth muscle cells and macrophages. ${ }^{23}$ Several studies have found that IL-18 is an important prognostic biomarker for atherosclerosis. ${ }^{24-6}$ In a multifactorial analysis that controlled for age and gender, a significant correlation was found between IL-18 and carotid intima-media thickness that was independent of traditional risk factors for atherosclerosis. ${ }^{27}$ It is postulated that IL-18 may contribute to the development of vascular diseases by inducing expression of IL-1-beta, TNF-alpha and NF-kappaB, inhibition of antiapoptosis factors (such as $\mathrm{Bcl}-2$ and $\mathrm{Bcl}-\mathrm{XL}$ ), upregulation of expression of pro-apoptosis factors (such as Fas, Fas-L and BclXS) and interference with mitogen-activated protein 
Table 2. Logistic Regression Analysis of Vascular Access Dysfunction According to Baseline Prognostic Markers

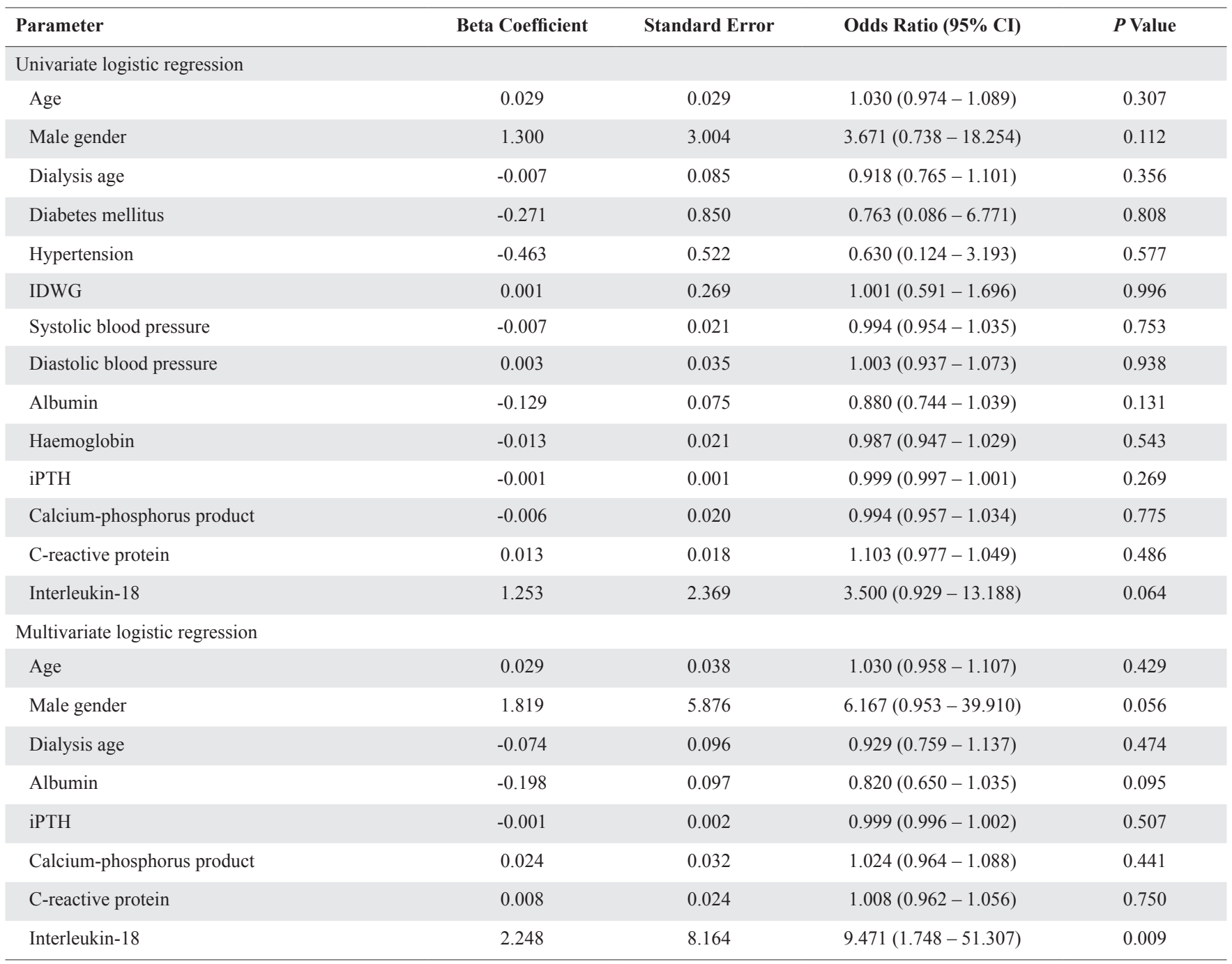

CI: Confidence interval; IDWG: Interval dialysis weight gain; iPTH: Immunoreactive parathyroid hormone

kinase-dependent signalling pathways. IL-18 may also promote proliferation and migration of vascular smooth muscle cells. ${ }^{28,29}$

While some studies had detected increased levels of serum IL-18 in CKD patients and those on CAPD, ${ }^{12}$ others found a correlation between elevated IL-18 and poor prognosis in HD patients including increased hospitalisation. ${ }^{15}$ Our study found that elevated IL18 was significantly correlated with VA dysfunction and this finding persisted even after adjustment had been made for age, gender, blood pressure, diabetes, dialysis age, ultrafiltration volume, anaemia, infection, secondary hyperparathyroidism, nutrition status and blood lipid level. These findings were also similar to those found for the correlation between IL-18 and atherosclerosis.

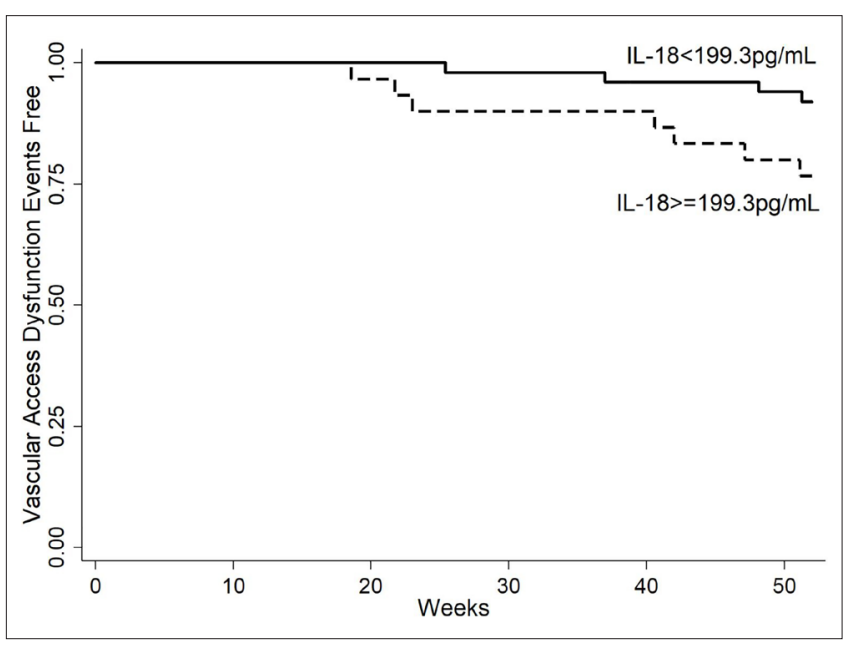

Fig. 1. Kaplan-Meier survival analysis showed that haemodialysis patients with high IL-18 $(\geq 199.3 \mathrm{pg} / \mathrm{mL})$ level had more vascular access dysfunction events $(P=0.047)$. 


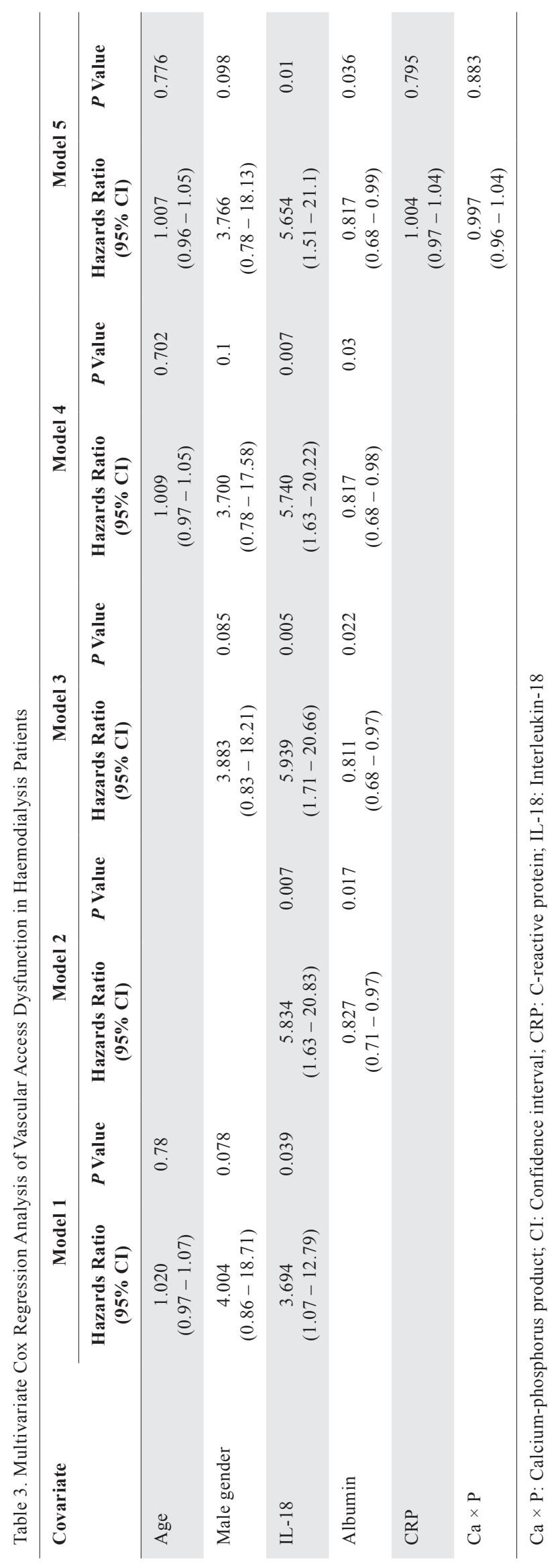

Our finding that elevated IL-18 and decreased albumin levels are independent risk factors for VA dysfunction, and that the combination of IL-18, albumin, age and gender can better predict risk of short-term VA dysfunction in HD patients, suggests that the causes of VA dysfunction are attributed to multiple factors.

Late AVF dysfunction is closely correlated with $\mathrm{NIH}$, and the contribution of IL-18 to AVF dysfunction requires further study. It is possible that IL-18 may have a role in the activation of the signal pathways described earlier, or it is involved in some causative mechanisms that are specific to ESRD patients.

Some of the limitations of our study included small sample size and short follow-up period. Consequently, our finding of a correlation between serum IL-18 level and VA dysfunction-and whether this correlation persists after a longer period of observation - may not be extrapolated to a larger HD population unless it is validated by further research. Also, the lack of regular ultrasound data on VA meant that our study recorded only adverse events that were related to access function in cases that required surgical intervention. Consequently, our results may be skewed. Finally, our study excluded IL-18 levels of healthy individuals from analysis.

\section{Conclusion}

This study has shown that serum IL-18 level is closely correlated with VA function in HD patients, and that elevated IL-18 can be used as an independent risk factor for short-term, adverse VA-related events in outpatients on HD. IL-18 can be used with serum albumin, age and gender to predict likelihood of such events in HD patients. Future cohort studies could involve larger sample sizes to validate our finding on the correlation between IL-18 and VA function. Further research is needed to elucidate how IL-18 contributes to VA dysfunction and new interventions that can improve access function and prognosis in HD patients. 
A

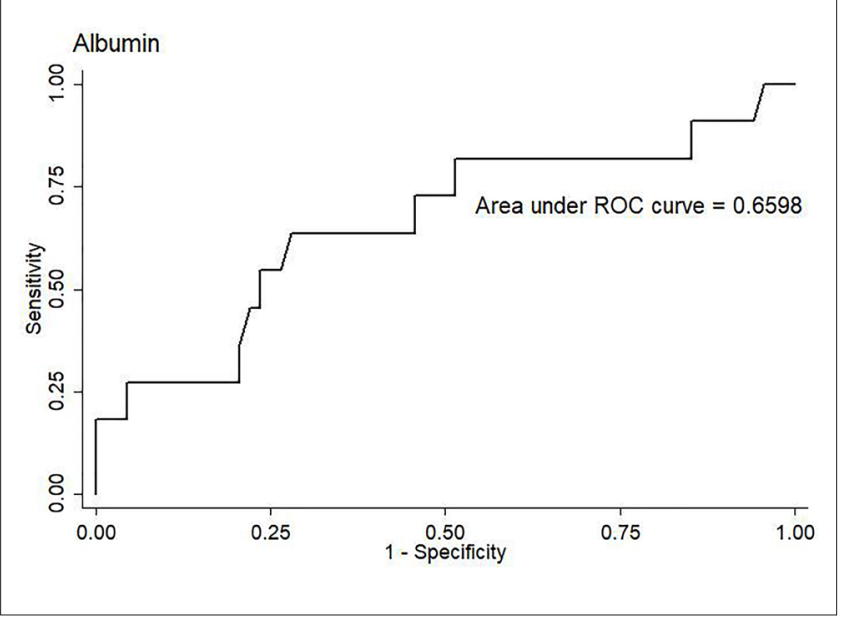

B

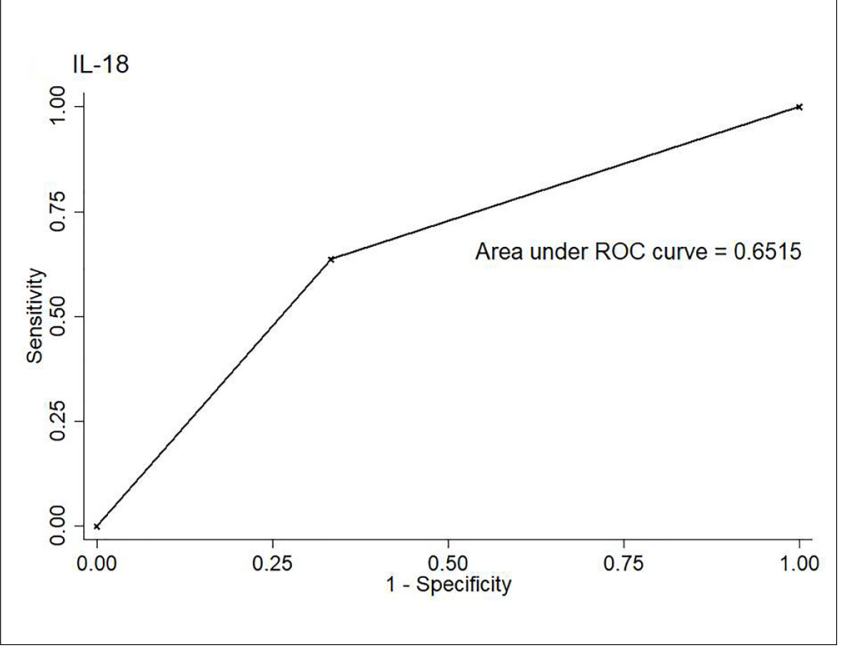

Fig. 2. Receiver operating characteristic (ROC) curve analysis of biochemical parameters in predicting vascular access dysfunction in haemodialysis patients. A: Serum albumin. B: Serum IL-18. C: Serum IL-18 with serum albumin. D: Serum IL-18 with serum albumin and gender. E: Serum IL-18 with serum albumin, gender and age. IL-18: Interleukin-18
C

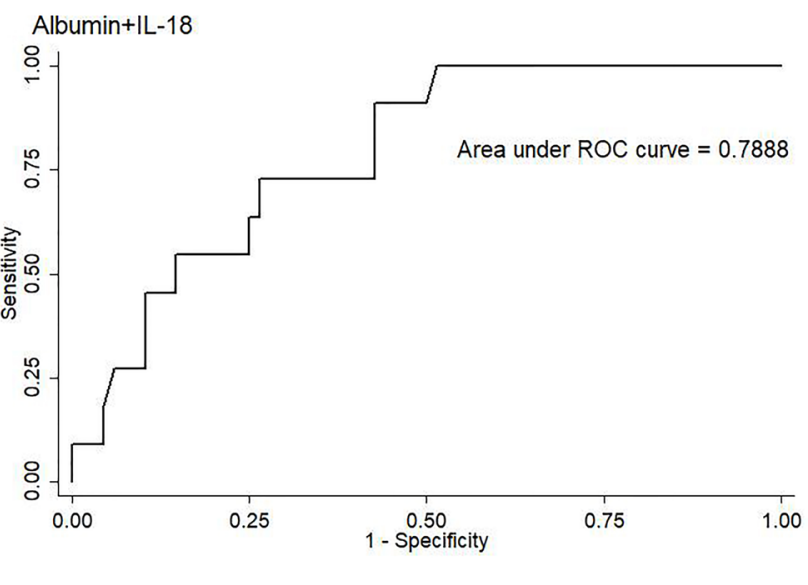

D

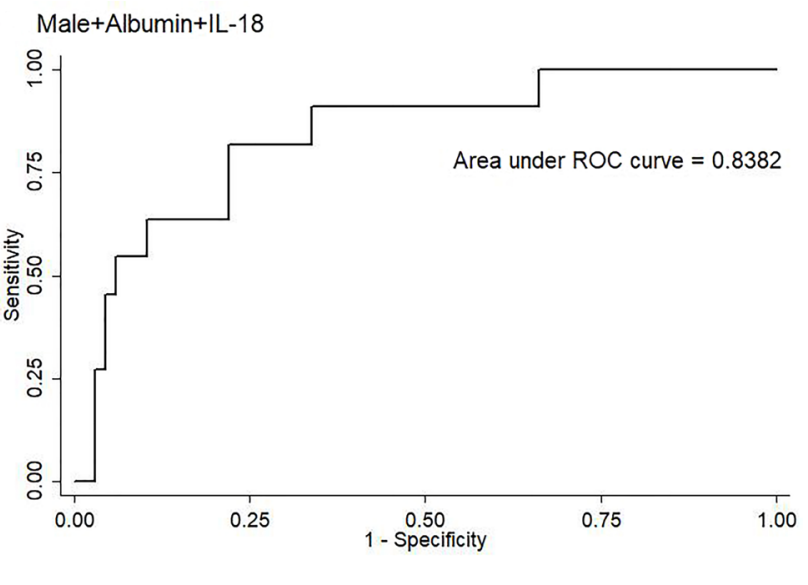

E

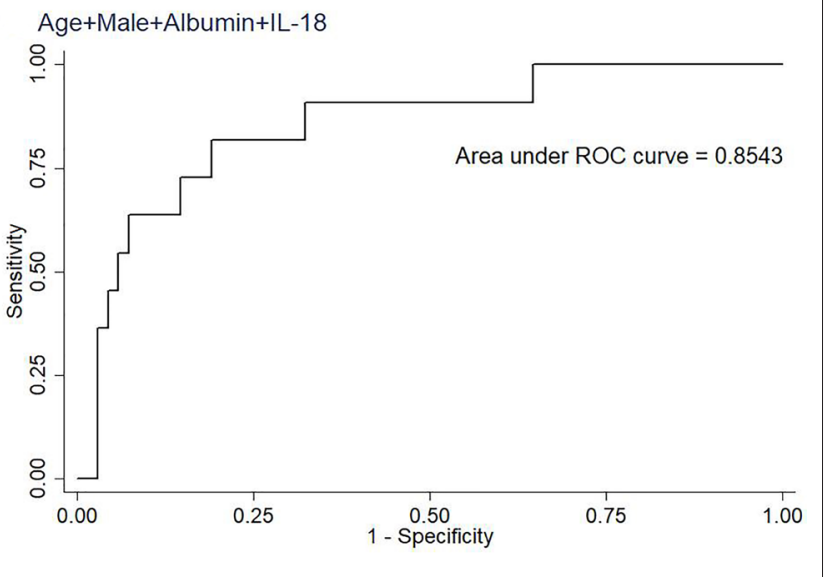




\section{REFERENCES}

1. Saran R, Robinson B, Abbott KC, Agodoa L, Bhave N, BraggGresham J, et al. US Renal Data System 2017 Annual Data Report: epidemiology of kidney disease in the United States. Am J Kidney Dis 2018;71:A7.

2. Ng TG, Tan SH. Novel trends in haemodialysis: where are we heading? Ann Acad Med Singapore 2010;39:482-8.

3. Ethier J, Mendelssohn DC, Elder SJ, Hasegawa T, Akizawa T, Akiba T, et al. Vascular access use and outcomes: an international perspective from the Dialysis Outcomes and Practice Patterns Study. Nephrol Dial Transplant 2008;23:3219-26.

4. Van Tricht I, De Wachter D, Tordoir J, Verdonck P. Hemodynamics and complications encountered with arteriovenous fistulas and grafts as vascular access for hemodialysis : a review. Ann Biomed Eng 2005;33:1142-57.

5. Roy-Chaudhury P, Spergel LM, Besarab A, Asif A, Ravani P. Biology of arteriovenous fistula failure. J Nephrol 2007;20:150-63.

6. Wahl P, Ducasa GM, Fornoni A. Systemic and renal lipids in kidney disease development and progression. Am J Physiol Renal Physiol 2016;310:F433-45.

7. Machowska A, Carrero JJ, Lindholm B, Stenvinkel P. Therapeutics targeting persistent inflammation in chronic kidney disease. Transl Res 2016;167:204-13.

8. Wasse H, Huang R, Naqvi N, Smith E, Wang D, Husain A. Inflammation, oxidation and venous neointimal hyperplasia precede vascular injury from AVF creation in CKD patients. $J$ Vasc Access 2012;13:168-74.

9. Liu BC, Li L, Gao M, Wang YL, Yu JR. Microinflammation is involved in the dysfunction of arteriovenous fistula in patients with maintenance hemodialysis . Chin Med J (Engl) 2008; $121: 2157-61$.

10. Wawrocki S, Druszczynska M, Kowalewicz-Kulbat M, Rudnicka W. Interleukin 18 (IL-18) as a target for immune intervention. Acta Biochim Pol 2016;63:59-63.

11. Xu Q, Tin SK, Sivalingam SP, Thumboo J, Koh DR, Fong KY. Interleukin-18 promoter gene polymorphisms in Chinese patients with systemic lupus erythematosus: association with CC genotype at position -607. Ann Acad Med Singapore 2007;36:91-5.

12. Chiang CK, Hsu SP, Pai MF, Peng YS, Ho TI, Liu SH, et al. Plasma interleukin-18 levels in chronic renal failure and continuous ambulatory peritoneal dialysis. Blood Purif 2005;23:144-8.

13. Formanowicz D, Wanic-Kossowska M, Pawliczak E, Radom M, Formanowicz P. Usefulness of serum interleukin-18 in predicting cardiovascular mortality in patients with chronic kidney diseasesystems and clinical approach. Sci Rep 2015;5:18332.

14. Wang S, Chen F, Yang S, Shi J. Interleukin-18. Int Heart J 2018;59:786-90.
15. Chiang CK, Hsu SP, Pai MF, Peng YS, Ho TI, Liu SH, et al. Interleukin-18 is a strong predictor of hospitalization in haemodialysis patients. Nephrol Dial Transplant 2004;19:2810-5.

16. Liu YW, Su CT, Chang YT, Tsai WC, Su YR, Wang SP, et al. Elevated serum interleukin-18 level is associated with all-cause mortality in stable hemodialysis patients independently of cardiac dysfunction. PLoS One 2014;9:e89457.

17. National Kidney Foundation. KDOQI Clinical Practice Guideline for Hemodialysis Adequacy: 2015 update. Am J Kidney Dis 2015;66:884-930.

18. Jin Q, Wang Y, Ye Z, Shi Y, Ma Z, Wang W, et al. Vascular access for haemodialysis consensus (version 1). Chinese J Blood Purif 2014;8:549-58.

19. Youden WJ. Index for rating diagnostic tests. Cancer 1950;3:32-5.

20. Vascular Access Work Group. Clinical practice guidelines for vascular access. Am J Kidney Dis 2006;48:S248-73.

21. Nath KA, Kanakiriya SK, Grande JP, Croatt AJ, Katusic ZS. Increased venous proinflammatory gene expression and intimal hyperplasia in an aorto-caval fistula model in the rat. Am J Pathol 2003;162:2079-90.

22. Gracie JA, Robertson SE, McInnes IB. Interleukin-18. J Leukoc Biol 2003;73:213-24.

23. Gerdes N, Sukhova GK, Libby P, Reynolds RS, Young JL, Schönbeck U. Expression of interleukin (IL)-18 and functional IL-18 receptor on human vascular endothelial cells, smooth muscle cells, and macrophages: implications for atherogenesis. J Exp Med 2002;195:245-57.

24. Packard RR, Libby P. Inflammation in atherosclerosis: from vascular biology to biomarker discovery and risk prediction. Clin Chem 2008;54:24-38.

25. Thompson SR, Humphries SE. Interleukin-18 genetics and inflammatory disease susceptibility. Genes Immun 2007;8:91-9.

26. Chandrasekar B, Mummidi S, Mahimainathan L, Patel DN, Bailey SR, Imam SZ, et al. Interleukin-18-induced human coronary artery smooth muscle cell migration is dependent on NF-kappaB- and AP-1-mediated matrix metalloproteinase-9 expression and is inhibited by atorvastatin. J Biol Chem 2006;281:15099-109.

27. Yamagami H, Kitagawa K, Hoshi T, Furukado S, Hougaku H, Nagai Y, et al. Associations of serum IL-18 levels with carotid intimamedia thickness. Arterioscler Thromb Vasc Biol 2005;25:1458-62.

28. Chandrasekar B, Vemula K, Surabhi RM, Li-Weber M, OwenSchaub LB, Jensen LE, et al. Activation of intrinsic and extrinsic proapoptotic signaling pathways in interleukin-18-mediated human cardiac endothelial cell death. J Biol Chem 2004;279:20221-33.

29. Chandrasekar B, Valente AJ, Freeman GL, Mahimainathan L, Mummidi S. Interleukin-18 induces human cardiac endothelial cell death via a novel signaling pathway involving NF-kappaB-dependent PTEN activation. Biochem Biophys Res Commun 2006;339:956-63. 